\title{
$L_{\text {loc }}^{2}$-BOUNDEDNESS FOR A CLASS OF SINGULAR FOURIER INTEGRAL OPERATORS
}

\author{
A. EL KOHEN
}

ABstract. We consider operators of the form $\int_{-\infty}^{\infty} F_{t} \phi(t) d t$ where $F_{t}$ is a 1-parameter family of Fourier integral operators and $\phi(t) d t$ a tempered distribution on the real line. We extend the result given in [1]. by

THEOREM. Let $F_{t}$ be a 1-parameter family of Fourier integral operators given

$$
F_{t} f(x)=\int_{\mathbf{R}^{n}} e^{i \phi(x, t, \theta)} a(x, t, \theta) \hat{f}(\theta) d \theta
$$

with $a$ and $\phi$ depending smoothly on $t$ and $a$ (resp. $\phi$ ) a symbol in the class $S_{1,0}^{0}$, i.e. $\operatorname{Sup}_{K}\left|\partial_{x}^{\alpha} \partial_{t}^{k} \partial_{\theta}^{\beta} a\right| \leq c_{K, \alpha, k, \beta}(1+|\theta|)^{-|\beta|}$ where $K$ is a compact set in $\mathbf{R}^{n} \times \mathbf{R}$ (resp. nondegenerate phase function) and $a(x, 0, \theta)=1($ resp. $\phi(x, 0, \theta)=x \cdot \theta)$. Furthermore, we assume that (i) for every $(x, \theta)$, finitely many of the vectors $v_{j}$, $j=1,2, \ldots$,

$$
v_{j}=\left.\frac{d^{j}}{d t^{j}} \phi_{\theta}(x, t, \theta)\right|_{t=0}
$$

generate $\mathbf{R}^{n}$, (ii) if $N=N_{x, \theta}$ is the first $j$ such that $v_{j} \cdot \theta \neq 0$ and $N \geq 5$, we assume the vectors $v_{1}, v_{2}, \ldots, v_{\nu}, 2 \nu<N \leq 2(\nu+1)$, all proportional. Then the operator $T=$ p.v. $\int_{-1}^{1} F_{t} d t / t$ is bounded from $L_{\text {comp }}^{2}\left(\mathbf{R}^{n}\right)$ to $L_{\text {loc }}^{2}\left(\mathbf{R}^{n}\right)$.

We have the following

COROLlaRY. Let $\gamma(x, t)$ be a variable curve of class $C^{\infty}$ in $\mathbf{R}^{n}, n=2$ or 3 , such that $\gamma(x, 0)=0$ and

(1) for $n=2$, the curves $t \rightarrow \gamma(x, t)$ have finite contact with their tangent lines at $t=0$,

(2) for $n=3$, the curves $t \rightarrow \gamma(x, t)$ have nonvanishing curvature and torsion at $t=0$.

Then the operator

$$
H f(x)=\text { p.v. } \int_{-1}^{1} f(x-\gamma(x, t)) \frac{d t}{t}
$$

is a bounded operator from $L_{\text {comp }}^{2}$ to $L_{\text {loc }}^{2}$.

When $n=2$ and the contact of the curves $t \rightarrow \gamma(x, t)$ with their tangent lines at $t=0$ equals 2 , i.e.: $\dot{\gamma} \times \ddot{\gamma} \neq 0$, then the $L^{2}$-estimate for the operator $H$ has been given in $[\mathbf{1}, \mathbf{3}]$.

Received by the editors March 7, 1983.

1980 Mathematics Subject Classification. Primary 35S99, 42B99.

Key words and phrases. Fourier transform, operator, singular integral.

(C) 1984 American Mathematical Society $0002-9939 / 84 \$ 1.00+\$ .25$ per page 
If we define

$$
\begin{aligned}
F_{t} f(x) & =f(x-\gamma(x, t)) \\
& =\int_{\mathbf{R}^{n}} \int_{\mathbf{R}^{n}} e^{i[(x-y) \theta-\gamma(x, t) \theta]} f(y) d y d \theta
\end{aligned}
$$

we see that we can use the Theorem above with $\phi(x, y, t, \theta)=(x-y) \theta-\gamma(x, t) \theta$ and $a \equiv 1$ to estimate the operator

$$
H=\text { p.v. } \int_{-1}^{1} F_{t} \frac{d t}{t} \text {. }
$$

The motivation for the estimates of such integrals can be shown using the method of rotation. In fact, a careful use of this method can reduce the estimate of many singular integrals over higher-dimensional surfaces to the estimate of singular integrals along curves.

In the statement of our result, condition (ii) insures that if a curve $\gamma(t)$ in $\mathbf{R}^{n}$ is flat in some direction, i.e.: $\gamma(t) \cdot \theta=0\left(t^{N}\right)$ near $t=0$ for some $\theta \in \mathbf{R}^{n}$, then the curve $\gamma(t)$ must be flat enough as a curve in $\mathbf{R}^{n}$.

The proof of our result follows from the asymptotic expansion of the function

$$
\omega=B \text { p.v. } \int_{-1}^{1} e^{i \phi(t)} a(t) \frac{d t}{t}
$$

where $\omega=\omega(x, \theta), \phi(t)=\phi(x, t, \theta), a(t)=a(x, t, \theta)$ and $B=B(\theta)$ is a smooth function on $\mathbf{R}^{n}$, identically zero near the origin and homogeneous of degree zero for $|\theta| \geq 1$.

We write the Taylor expansion

$$
\phi(t)=x \cdot \theta+t \sigma_{1}+\cdots+\frac{t^{N-1}}{(N-1) !} \sigma_{N-1}+\frac{t^{N}}{N !} \sigma_{N}(t)
$$

where

$$
\sigma_{j}=\left.\frac{d^{j}}{d t^{j}} \phi(x, t, \theta)\right|_{t=0}, \quad j=1,2, \ldots
$$

From the hypothesis, for any $\left(x_{0}, \theta_{0}\right), \theta_{0} \neq 0$, there exists $N \geq 1$ and a conic neighborhood $K \times \Gamma$ of $\left(x_{0}, \theta_{0}\right)$ on which $\left|\sigma_{N}(x, \theta)\right| \geq a|\theta|$ and such that $\sigma_{1}=$ $\sigma_{2}=\cdots=\sigma_{N-1}=0$ at $\left(x_{0}, \theta_{0}\right)$. The case $N=1$ or 2 is given in [1]. We may, then, assume $N \geq 3$ and restrict our attention to the following direction: $\Gamma_{N}=\left\{\theta \in \mathbf{R}^{n}: \sigma_{N}(x, \theta) \geq a|\theta|\right.$ for all $\left.x \in K\right\}$.

The direction $-\Gamma_{N}$ can be considered in a similar fashion.

LEMMA 1. The operator corresponding to the symbol

$$
\omega_{1}=B \text { p.v. } \int_{|t| \leq 1 / \sqrt[N]{\sigma_{N}}} e^{i t \sigma_{1}+\cdots+i t^{N} / N ! \sigma_{N}(t)} a(t) \frac{d t}{t}
$$

is bounded from $L_{\text {comp }}^{2}$ to $L_{\text {loc }}^{2}$.

ProOF. For simplicity we will write $1 / \sqrt[N]{\sigma_{N}}=q$; we then have

$$
\begin{aligned}
\omega_{1} & =\int_{|t| \leq q} e^{i t \sigma_{1}+\cdots+i t^{N} / N ! \sigma_{N}} \frac{d t}{t}+E_{1} \\
& =\int_{|t| \leq 1} e^{i t q \sigma_{1}+\cdots+i t^{N-1} /(N-1) ! q^{N-1} \sigma_{N-1}} e^{i t^{N} / N !} \frac{d t}{t}+E_{1}
\end{aligned}
$$


where

$$
\begin{gathered}
E_{1}=\int_{|t| \leq q} e^{i t \sigma_{1}+\cdots+i t^{N} / N ! \sigma_{N}}\left(e^{i t^{N}\left(\sigma_{N}(t)-\sigma_{N}\right) / N !} a(t)-1\right) \frac{d t}{t} \\
=\int_{|t| \leq 1} e^{i t q \sigma_{1}+\cdots+i t^{N-1} q^{N-1} \sigma_{N-1} /(N-1) !} \\
\quad \times\left(e^{i t^{N}\left(\sigma_{N}(t q) / \sigma_{N}-1\right) / N !} a(t q)-1\right) e^{i t^{N} / N !} \frac{d t}{t} .
\end{gathered}
$$

For each $t,|t| \leq 1$, the operator corresponding to the integrand in $E_{1}{ }^{1}$ is a Fourier integral operator with phase function

$$
x \cdot \theta+t q \sigma_{1}+\cdots+\frac{1}{\nu !} t^{\nu} q^{\nu} \sigma_{\nu}, \quad \nu<N \leq 2(\nu+1)
$$

which is clearly nondegenerate for large $\theta$, i.e. $B(\theta) \neq 0$ (uniformly in $t,|t| \leq 1$ ), since $q$ is homogenous of degree $-1 / N$ and $\sigma_{1}, \sigma_{2}, \ldots, \sigma_{\nu}$ of degree 1 and whose amplitude is

$$
e^{i t^{\nu+1} \cdot q^{\nu+1} \sigma_{\nu+1} /(\nu+1) !+\cdots+i t^{N-1} q^{N-1} \sigma_{N-1} /(N-1) !} \frac{1}{t}\left(e^{i t^{N}\left(\sigma_{N}(t q) / \sigma_{N}-1\right) / N !} a(t q)-1\right)
$$

easily seen to be in $S_{(\nu+1) / N, 1-(\nu+1) / N}^{-1 / N}\left(K \times \Gamma_{N}\right)$. Notice that $q$ belongs to $S_{1,1 / N}^{-1 / N}\left(K \times \Gamma_{N}\right)$.

The operator corresponding to $E_{1}$ thus verifies the conclusion of Lemma 1.

We next write

$$
\omega_{1}-E_{1}=B \text { p.v. } \int_{|t| \leq 1} e^{i t q \sigma_{1}+\cdots+i t^{\nu} q^{\nu} \sigma_{\nu} / \nu !} \frac{d t}{t}+E_{2} .
$$

The operator corresponding to $E_{2}$ is an absolutely convergent integral of Fourier integral operator with phase functions the same as for $E_{1}$ and amplitudes in $S_{(\nu+1) / N, 1-(\nu+1) / N}^{0}\left(K \times \Gamma_{N}\right)$, and hence verifies the conclusion of Lemma 1 .

Now, for $\Omega_{1}=\omega_{1}-E_{1}-E_{2}$, we use condition (ii) (in case $N \geq 5$ ) to write

$$
\Omega_{1}=B \text { p.w. } \int_{|t| \leq 1} e^{i t q \sigma_{1} \phi(t)} \frac{d t}{t}
$$

where

$$
\phi(t)=1+\frac{t}{2 !} q \frac{\sigma_{2}}{\sigma_{1}}+\cdots+\frac{t^{\nu-1}}{\nu !} q^{\nu-1} \frac{\sigma_{\nu}}{\sigma_{1}} .
$$

We notice that condition (ii) implies that $\sigma^{2} / \sigma_{1}, \ldots, \sigma_{\nu} / \sigma_{1}$ belong to $S_{1,0}^{0}\left(K \times \Gamma_{N}\right)$. We, then, let $s=t \phi(t)$ and choose $b>0$ small enough so that $s(t)$ is strictly increasing on $[-b, b]$ uniformly on $K \times \Gamma_{N}$ since $s(0)=0, s^{\prime}(0)=1$ and $\phi(t)=$ $1+O(t)$ uniformly on $K \times \Gamma_{N}$. We now write

$$
\Omega_{1}=B \text { p.v. } \int_{|t| \leq b} e^{i t q \sigma_{1} \phi(t)} \frac{d t}{t}+F_{1} .
$$

The operator corresponding to $F_{1}$ is an absolutely convergent integral of Fourier integral operators with phase functions $x \cdot \theta+t q \sigma_{1} \phi(t), b \leq|t| \leq 1$ and hence verifies the conclusion of Lemma 1.

\footnotetext{
${ }^{1}$ Strictly speaking, the operator corresponding to the integrand in $E_{1}$ is not a Fourier integral operator because the phase is not homogeneous of degree 1 . However, this does not change our conclusion. The same remark is valid for the operators corresponding to $E_{2}$ and $F_{1}$.
} 
We now use the change of variable $s=t \phi(t)$ to write

$$
\begin{aligned}
\Omega_{1}-F_{1} & =B \text { p.v. } \int_{|t| \leq b} e^{i t q \sigma_{1} \phi(t)} \frac{d t}{t} \\
& =B \text { p.v. } \int_{-b \phi(-b)}^{b \phi(b)} e^{i s q \sigma_{1}} g(s) \frac{d s}{s}
\end{aligned}
$$

where $g(s)=1 /\left(1+s \phi^{\prime} / \phi\right)$ and $\phi^{\prime} / \phi=\frac{1}{2} q \sigma_{2} / \sigma_{1}$ at $s=0$. Hence

$$
g(s)=1-\frac{s}{2} q \frac{\sigma_{2}}{\sigma_{1}}+\cdots
$$

and

$$
\Omega_{1}-F_{1}=B \text { p.v. } \int_{-b \phi(-b)}^{b \phi(b)} e^{i s q \sigma_{1}} \frac{d s}{s}-\frac{B}{2} \frac{\sigma_{2}}{\sigma_{1}} q \int_{-b \phi(-b)}^{b \phi(b)} e^{i s q \sigma_{1}} d s+\cdots
$$

Since $\sigma_{2} q / \sigma_{1}$ is of negative order and $\sigma_{2} q / \sigma_{1}$ belongs to $S_{1,1 / N}^{-1 / N}\left(K \times \Gamma_{N}\right)$, we need only estimate

$$
Q_{j}(x, \theta)=\int_{-b \phi(-b)}^{b \phi(b)} e^{i s q \sigma_{1}} s^{j} d s
$$

for $j=-1,0,1,2, \ldots$

Estimate of $Q_{-1}$. We write

$$
\begin{aligned}
Q_{-1} & =\text { p.v. } \int_{-b \phi(b)}^{b \phi(b)} e^{i s q \sigma_{1}} \frac{d s}{s}-\int_{-b \phi(b)}^{-b \phi(-b)} e^{i s q \sigma_{1}} \frac{d s}{s} \\
& =Q_{-1}^{1}-Q_{-1}^{2} .
\end{aligned}
$$

For $Q_{-1}^{1}$ we write

$$
\begin{aligned}
Q_{-1}^{1} & =\text { p.v. } \int_{-b}^{b} e^{i s q \sigma_{1} \phi(b)} \frac{d s}{s} \\
& =\int_{-\infty}^{\infty} e^{i s q \sigma_{1} \phi(b)} \frac{\psi(s)}{s} d s+G_{1}
\end{aligned}
$$

where $\psi$ is a smooth function with compact support, $\psi \equiv 1$ near 0 and the operator corresponding to $G_{1}$ an absolutely convergent integral of Fourier integral operators. We choose $\psi$ even and such that

$$
\left|\hat{\psi}^{(j)}(x)\right| \leq 3^{j} e^{-2 x}, \quad j \geq 0
$$

for $x>0$. We next choose $c>0$ such that $\sigma_{1}(x, \theta)+c|\theta| \geq 2 c|\theta| / 3$ on $K \times \Gamma_{N}$ since $\sigma_{1}\left(x_{0}, \theta_{0}\right)=0$; we then write

$$
\begin{aligned}
Q_{-1}^{1}-G_{1} & =\left(\frac{\psi}{s}\right) \widehat{\left(q \sigma_{1} \phi(b)\right)} \\
& =\sum_{k \geq 0} \frac{(-c)^{k} q^{k} \phi(b)^{k}|\theta|^{k}}{k !} \hat{\psi}^{(k-1)}\left(q\left(\sigma_{1}+c|\theta|\right)\right) \\
& =\left(\frac{\psi}{s}\right) \hat{(}\left(q\left(\sigma_{1}+c|\theta|\right)\right)+\sum_{k \geq 1} \frac{(-c)^{k}}{k !} \rho_{k}
\end{aligned}
$$


with

$$
\rho_{k}=q^{k} \phi(b)^{k}|\theta|^{k} \hat{\psi}^{(k-1)}\left(q\left(\sigma_{1}+c|\theta|\right)\right) .
$$

It is easily seen that $(\psi / s)^{\uparrow}\left(q\left(\sigma_{1}+c|\theta|\right)\right)$ belongs to $S_{1,0}^{0}\left(K \times \Gamma_{N}\right)$ since $(\psi / s)^{-}$ is bounded, $(d / d u)(\psi / s) \widehat{\Upsilon}(u)$ rapidly decreasing and $q\left(\sigma_{1}+c|\theta|\right)$ elliptic of order $1-1 / N$ on $K \times \Gamma_{N}$, and $\rho_{k}$ belongs to $S_{1,0}^{-\infty}\left(K \times \Gamma_{N}\right)$ for all $k \geq 1$. We then need only estimate

$$
R_{1}=\sum_{k \geq 1} \frac{(-c)^{k}}{k !} \rho_{k}
$$

But if we write $R_{1}=\bar{R}_{1} /|\theta|^{M}, M>0$, then $\bar{R}_{1}$ is easily seen to be bounded by a constant depending only on $c$ and $M$ uniformly on $K \times \Gamma_{N}$. If we choose $M>n / 2$, the rest of the estimate is obvious since we only seek local estimates.

For $Q_{-1}^{2}$, we use a change of variable to write

$$
\begin{aligned}
Q_{-1}^{2}= & e^{-b q \sigma_{1} \phi(b)}\left(1-\frac{\phi(-b)}{\phi(b)}\right) \int_{0}^{1} e^{i s b q(\phi(b)-\phi(-b))} \frac{d s}{(1-\phi(-b) / \phi(b)) s-1} \\
= & \left(1-\frac{\phi(-b)}{\phi(b)}\right) \int_{0}^{1} e^{-i s b q \sigma_{1} \phi(-b)} d s \\
& -\left(1-\frac{\phi(-b)}{\phi(b)}\right)^{2} \int_{0}^{1} e^{-s b q \sigma_{1} \phi(-b)} s d s+\cdots \\
= & q \frac{\sigma_{2}}{\sigma_{1}} \int_{0}^{1} e^{-i s b q \sigma_{1} \phi(-b)} d s-\left(q \frac{\sigma_{2}}{\sigma_{1}}\right)^{2} \int_{0}^{1} e^{-i s b q \sigma_{1} \phi(-b)} s d s+\cdots
\end{aligned}
$$

Notice that only finitely many of the terms in this expansion are needed since $q \sigma_{2} / \sigma_{1}$ is of negative order and all the integrals are absolutely convergent. Also, clearly, the operator corresponding to each term in the expression verifies the conclusion of Lemma 1.

Estimate for $Q_{j}, j \geq 0$. For $j \geq 0$, the operator corresponding to

$$
Q_{j}=\int_{-b \phi(-b)}^{b \phi(b)} e^{i q \sigma_{1} s} s^{j} d s
$$

is easily seen to verify the conclusion of Lemma 1, using the same arguments as for the operator corresponding to $Q_{-1}^{2}$.

LEMMA 2. The operator corresponding to

$$
\omega_{2}=B \text { p.v. } \int_{q \leq|t| \leq 1} e^{i \phi(t)} a(t) \frac{d t}{t}, \quad q=\frac{1}{\sqrt[N]{\sigma_{N}}},
$$

is bounded from $L_{\text {comp }}^{2}$ to $L_{\text {loc }}^{2}$.

Proof. We let $t(x, \theta)$ be such that $(d / d t) \phi(x, t(x, \theta), \theta)=0$. Clearly $t(x, \theta)$ is homogeneous of degree 0 in $\theta$. Moreover, if $q \leq|t(x, \theta)| \leq 1$ then $t(x, \theta)$ necessarily stays away from 0 on $K \times \Gamma_{N}$. We then write

$$
\omega_{2}=B \text { p.v. } \int_{-\infty}^{\infty} e^{i \phi(t)} a(t) \frac{\psi(t)}{t} d t+G_{2}
$$


where $\psi$ is a smooth function and

$$
\left\{t(x, \theta):(x, \theta) \in K \times \Gamma_{N}\right\} \subset \operatorname{supp} \psi \subset(0,1] .
$$

For $G_{2}$ we use integration by parts to show that the corresponding operator is a Fourier integral operator with amplitude in $S_{1,0}^{-1}$. The other term is clearly an absolutely convergent integral; hence the corresponding operator is an absolutely convergent integral of Fourier integral operators which finishes the proof of Lemma 2.

The proof of the Theorem follows from the two lemmas and the use of a microlocal partition of unity associated with the different directions and a neighborhood of the origin. Notice that

$$
\begin{aligned}
E_{0}(x, \theta) & =(1-B(\theta)) \text { p.v. } \int_{-1}^{1} e^{i \phi(x, t, \theta)} a(x, t, \theta) \frac{d t}{t} \\
& =(1-B(\theta)) \int_{-1}^{1}\left(e^{i \phi(x, t, \theta)} a(x, t, \theta)-e^{i x \cdot \theta}\right) \frac{d t}{t}
\end{aligned}
$$

is a symbol of a smoothing operator.

\section{REFERENCES}

1. A. El Kohen, On Fourier integral operators, Proc. Amer. Math. Soc. 85 (1982), 567-571.

2. F. Treves, Pseudo-differential and Fourier integral operators, vols. 1 and 2, The Univ. Ser. in Math., Plenum Press, New York, 1980.

3. S. Wainger and G. Weiss (eds.), Proc. Sympos. Pure Math., vol. 35, Part 1, Amer. Math. Soc., Providence, R.I., 1979.

Department of Mathematics, Texas A\&M University, College Station, TEXAS 77843

Current address: Faculté des Sciences, Département de Mathématiques, B.P. 1796 Atlas, Fes, Morocco 